\title{
Endoscopic "crossroads" in the management of malignant hilar strictures
}

\author{
Shajan Peter • Charles M. Wilcox
}

Received: 8 October 2013 / Accepted: 8 October 2013 /Published online: 26 October 2013

(C) Indian Society of Gastroenterology 2013

Malignant biliary strictures can result from primary or metastatic disease processes of the intrahepatic or extrahepatic duct(s) [1]. Hilar cholangiocarcinomas are the most common type of primary extrahepatic bile duct cancers occurring at or near the junction of the right and left hepatic ducts or common hepatic duct. The modified Bismuth-Corlett system classifies such tumor into four types based on the extent of these lesions (types 1-4). These can present with symptoms of biliary obstruction, and accurate diagnosis and staging are needed for further management. The majority of hilar lesions are advanced and unresectable at the time of diagnosis, requiring palliative management. Palliative biliary drainage is effective in relieving obstructive symptoms, improving quality of life though there is no benefit on survival [2]. An endoscopic approach may be preferred to surgical or percutaneous methods and depends on the extent of the tumor and operability of the patient.

The choice on the best targeted, optimal endoscopic decompression method needed for prolonged symptom-free survival associated with low procedural related complications and fewer hospital days is still not clear. Placement of endoscopic biliary stents remains the primary decompressive technique of choice. Further determination of appropriate therapeutic strategy such as plastic vs. self-expanding metal stents (SEMS), unilateral vs. bilateral, is influenced by several factors such as stricture length and extent, imaging findings, expected patient survival, potential need for removability, and cost [3, 4].

Stenting for malignant hilar strictures (MHS) can be technically challenging due to the complex anatomy. Studies have shown that SEMS outperformed plastic stents allowing higher rates of successful drainage, prolonged patency, and lower

\footnotetext{
S. Peter · C. M. Wilcox $(\bowtie)$

Department of Gastroenterology, Basil Hirschowitz Endoscopic Center of Endoscopic Excellence, University of Alabama at Birmingham, 6th Floor Jefferson Tower, 625 19th Street South, Birmingham, AL 35249, USA

e-mail:melw@uab.edu
}

adverse outcomes such as cholangitis, stent migration, etc. $[5,6]$. The adequacy of unilateral or bilateral drainage continues to be debated $[7,8]$. A study published in this issue of the Journal by Puli et al. [9] addresses the role of unilateral and bilateral stenting for MHS. In a pooled systematic analysis, they showed that bilateral SEMS had a lower odds for overall complications and higher odds for lowering bilirubin levels when comparing studies using unilateral stenting. Placement in either one or both systems, however, did not change the 30-day mortality rates. When analyzing plastic stenting separately, unilateral stenting was comparable to bilateral stenting in terms of success, complications, cholangitis, and 30-day mortality. The study has limitations as the analysis included nonrandomized observational studies. In addition, the exact Bismuth classification for all studies was lacking, and the outcomes were heterogeneous.

This study echoes the results of another recent systematic review by Hong et al. [10]. Stent insertion rate was higher with the unilateral treatment group compared to bilateral drainage group, while no difference was observed between groups with respect to successful drainage, early complications, stent patency, and patient survival. In the same analysis, compared with plastic stents, the use of SEMS was associated with higher successful drainage rate, lower early complication rate, and longer stent patency.

What have we learned from these studies? Firstly, endoscopic palliative drainage for malignant biliary obstruction is preferred; however, careful reviewing of the clinical presentation, anatomy, and location is necessary. Higher-grade strictures such as Bismuth type $>2$ can be a challenge, and management depends on the expertise and available radiological backup as a combination of percutaneous and endoscopic approach may be required in certain cases [11]. Secondly, preprocedural strategic planning with adequate imaging is important before any attempted endoscopic treatment. Both magnetic resonance imaging/magnetic resonance cholangiopancreatography and 
multidetector computed tomography scan are useful for road mapping while assessing the level of obstruction and measurement of dominant liver sectors and providing necessary ductal and parenchymal information such as liver volumes and lobe atrophy $[12,13]$. Thirdly, one has to decide on the choice of stents. Either plastic or SEMS provide short-term benefits with regards to success (decrease in jaundice, serum bilirubin, or pruritus) and improvement in quality of life. Plastic stents have advantages of being less expensive, technically easy to insert, and can be removed and exchanged; however, these have the downside of limited stent patency ( $\sim 3$ months). On the other hand, SEMS offer better drainage and have a longer stent patency ( $\sim 9$ months). In the presence of SEMS, chemoradiation can be simultaneously given, and trans-stent photodynamic therapy can also be performed with dose adjustments. In short, SEMS are preferred but will depend on the expertise available and the "life expectancy" of the patient. Finally, the question of draining one or more segments remains. Selection of the liver sector and ductal system should be decided with the aim to drain $>50 \%$ of liver volume which is a good predictor for longer survival [13]. As an arbitrary measure, approximately $55 \%$ to $60 \%$ of liver volume is drained via the right hepatic duct, $30 \%$ to $35 \%$ by the left hepatic duct, and $10 \%$ from the caudate lobe. Careful cannulation of the targeted system, avoiding contamination of the other (eg. atrophic segment), is needed as the goal should be to drain an intentionally opacified system. With this in mind, unilateral stenting of the dominant segment may be sufficient for the resolution of symptoms in many patients. Nevertheless, bilateral stenting may be required for preserving functional reserve volume or in a situation where inadvertent contrast has been injected to avoid the risk of cholangitis. The feasibility of deploying bilateral uncovered SEMS will depend on individual expertise in understanding the technical challenges, especially in high-grade Bismuth type 3 or 4 MHS. Some of these include prudent antibiotic coverage, consideration for dilatation given the tight and angulated nature of some lesions, minimizing contrast injection, as well as exact positioning of stents with avoidance of a "biliary cripple" (distal end of one abutting the proximal end of other) while bearing in mind that reintervention may be difficult.

In case of stent dysfunction, occlusions (debris or tumor in/ overgrowth), or migration, plastic stents are removed and replaced. Uncovered SEMS have a lower reintervention rate and can be cleaned with newer stent-in-stent insertions (either plastic or metal). The overall success for endoscopic revisions varies from $45 \%$ to $100 \%$. The success rate for endoscopic reintervention is higher with unilateral than bilateral stenting ( $100 \%$ vs. $68 \%$ ) [14]. The role of preoperative biliary decompression is not clear and may be beneficial if there is a delay in surgical resection or requiring neoadjuvant therapy.

With growing expertise and better imaging techniques, current endoscopic management of MHS has evolved remarkably over the last few years. Once considered a difficult frontier to cross, with advancement of technology, newer stent designs, a multisegment approach is achievable, facilitating adequate drainage minimizing risk of complications. Selective sectorial upstream cannulation still remains a challenge, and technical failure to bridge can nevertheless occur in up to $20 \%$ of cases; therefore, involvement of a multidisciplinary experienced team may be necessary. Recent advancement in areas such as cholangioscopy and intraductal endoscopic ultrasound will refine future endoscopic diagnosis and better accessibility of these lesions. All these developments reinforce the need for larger powered multicentric randomized trials to define clear predictors and outcomes for adequate drainage as well as management strategies. Such studies will further our knowledge and provide better guidelines for a judicious algorithmic model for endoscopic therapy of MHS.

\section{References}

1. National Comprehensive Cancer Network (2013) NCCN guidelines version 2. Extrahepatic cholangiocarcinoma. NCCN.org. http://www. nccn.org/professionals/physician_gls/pdf/hepatobiliary.pdf. Accessed 1 Oct 2013.

2. Larghi A, Tringali A, Lecca PG, Giordano M, Costamagna G. Management of hilar biliary strictures. Am J Gastroenterol. 2008;103: 458-73.

3. Rerknimitr R, Angsuwatcharakon P, Ratanachu-ek T, et al. AsiaPacific consensus recommendations for endoscopic and interventional management of hilar cholangiocarcinoma. J Gastroenterol Hepatol. 2013;28:593-607.

4. Dumonceau JM, Tringali A, Blero D, et al. Biliary stenting: indications, choice of stents and results: European Society of Gastrointestinal Endoscopy (ESGE) clinical guideline. Endoscopy. 2012;44:277-98.

5. Perdue DG, Freeman ML, DiSario JA, et al. Plastic versus selfexpanding metallic stents for malignant hilar biliary obstruction: a prospective multicenter observational cohort study. J Clin Gastroenterol. 2008;42:1040-6.

6. Wagner HJ, Knyrim K, Vakil N, Klose KJ. Plastic endoprostheses versus metal stents in the palliative treatment of malignant hilar biliary obstruction. A prospective and randomized trial. Endoscopy. 1993;25:213-8.

7. De Palma GD, Galloro G, Siciliano S, Iovino P, Catanzano C. Unilateral versus bilateral endoscopic hepatic duct drainage in patients with malignant hilar biliary obstruction: results of a prospective, randomized, and controlled study. Gastrointest Endosc. 2001;53: $547-53$.

8. Webb K, Saunders M. Endoscopic management of malignant bile duct strictures. Gastrointest Endosc Clin N Am. 2013;23: 313-31.

9. Puli SR, Kalva N, Pamulaparthy SR, et al. Bilateral and unilateral stenting for malignant hilar obstruction: a systematic review and meta-analysis. Indian J Gastroenterol. 2013; 32. doi:10.1007/ s12664-013-0413-3.

10. Hong W, Sun X, Zhu Q. Endoscopic stenting for malignant hilar biliary obstruction: should it be metal or plastic and unilateral or bilateral? Eur J Gastroenterol Hepatol. 2013. doi:10.1097/MEG. 0b013e328360b9ec. 
11. Dowsett JF, Vaira D, Hatfield AR, et al. Endoscopic biliary therapy using the combined percutaneous and endoscopic technique. Gastroenterology. 1989;96:1180-6.

12. Akamatsu N, Sugawara Y, Osada H, et al. Diagnostic accuracy of multidetector-row computed tomography for hilar cholangiocarcinoma. J Gastroenterol Hepatol. 2010;25:731-7.
13. Vienne A, Hobeika E, Gouya H, et al. Prediction of drainage effectiveness during endoscopic stenting of malignant hilar strictures: the role of liver volume assessment. Gastrointest Endosc. 2010;72:728-35.

14. Yasuda I, Mukai T, Moriwaki H. Unilateral versus bilateral endoscopic biliary stenting for malignant hilar biliary strictures. Dig Endosc. 2013;25 Suppl 2:81-5. 